\title{
Association Between $N$-Desmethylclozapine and Clozapine-Induced Sialorrhea: Involvement of Increased Nocturnal Salivary Secretion via Muscarinic Receptors by $N$-Desmethylclozapine ${ }^{\mathbb{S}}$
}

\author{
Shuhei Ishikawa, ${ }^{1}$ Masaki Kobayashi, Naoki Hashimoto, Hideaki Mikami, Akihiko Tanimura, \\ Katsuya Narumi, Ayako Furugen, Ichiro Kusumi, and Ken Iseki \\ Laboratory of Clinical Pharmaceutics and Therapeutics, Division of Pharmasciences, Faculty of Pharmaceutical Sciences (S.I., \\ M.K., H.M., K.N., A.F., K.I.) and Education Research Center for Clinical Pharmacy, Faculty of Pharmaceutical Sciences (M.K.), \\ Hokkaido University, Sapporo, Japan; Department of Pharmacy, Hokkaido University Hospital, Sapporo, Japan (S.I.); Department \\ of Psychiatry, Hokkaido University Graduate School of Medicine, Sapporo, Japan (N.H., I.K.); and Department of Pharmacology, \\ School of Dentistry, Health Sciences University of Hokkaido, Sapporo, Japan (A.T.)
}

Received June 11, 2020; accepted August 12, 2020

\begin{abstract}
Clozapine-induced sialorrhea (CIS) is a common side effect of clozapine. There is no established standard treatment of CIS since the underlying mechanism remains unknown. This study aimed to elucidate the mechanisms involved in CIS. In our clinical study, a prospective observational study evaluated the association between serum and saliva concentrations of clozapine or its metabolites and Drooling Severity and Frequency Scale (DSFS) score. In our in vivo study, we first developed a new CIS animal model; subsequently, we measured salivary secretion and concentrations of clozapine or its metabolites in the animal model. In our in vitro study, we measured the calcium ion $\left(\mathrm{Ca}^{2+}\right)$ response to evaluate the effect of clozapine or its metabolites on human salivary gland cell line (HSY cells) and then examined whether their effect was inhibited by atropine. In our clinical study, serum and saliva $N$-desmethylclozapine concentrations were significantly correlated with nocturnal DSFS score. In our in vivo study, daily single oral administration of $100 \mathrm{mg} / \mathrm{kg}$ clozapine for 7 days significantly increased salivary secretion in rats. Furthermore, $\mathrm{N}$-desmethylclozapine concentrations in serum and
\end{abstract}

submandibular glands of the rats were higher than clozapine concentrations. In our in vitro study, $\mathrm{N}$-desmethylclozapine only elicited an increase in the intracellular $\mathrm{Ca}^{2+}$ in HSY cells. $\mathrm{N}$-desmethylclozapine-induced $\mathrm{Ca}^{2+}$ responses were inhibited by atropine. These results suggest that $N$-desmethylclozapine is implicated in CIS by increasing nocturnal salivation via the muscarinic receptors. Moreover, our developed animal model that reflects $\mathrm{CIS}$ in clinical condition plays a key role as a bridge between basic and clinical research.

\section{SIGNIFICANCE STATEMENT}

Clozapine-induced sialorrhea (CIS) is a severe and frequent adverse reaction, but the mechanism underlying CIS is less well understood. This paper reports that $N$-desmethylclozapine, a metabolite of clozapine, is implicated in CIS by increasing nocturnal salivation via the muscarinic receptors and that oral administration of clozapine at $100 \mathrm{mg} / \mathrm{kg}$ once daily for 7 days to rat is the optimum method for establishing the new animal model reflecting the clinical scenario of CIS.
This study was supported by the Japan Society for the Promotion of Science (JSPS) [KAKENHI Grant Numbers 15H00540, 16H00509, and 19H0035409] and Takeda Science Foundation.

Conflict of interests: S.I., M.K., H.M., A.T., K.N., A.F., and K.I. declare that they have no conflicts of interest with the contents of this article. N.H. received personal fees from Janssen Pharmaceutical, Yoshitomiyakuhin, Otsuka Pharmaceutical, Dainippon Sumitomo Pharma, Novartis Pharma, and Meiji Seika Pharma. I.K. has received honoraria from Astellas, Daiichi Sankyo, Dainippon Sumitomo Pharma, Eisai, Eli Lilly, Janssen Pharmaceutical, Kyowa Hakko Kirin, Lundbeck, Meiji Seika Pharma, MSD, Mylan, Novartis Pharma, Ono Pharmaceutical, Otsuka Pharmaceutical, Pfizer, Shionogi, Shire, Taisho Toyama Pharmaceutical, Takeda Pharmaceutical, Tanabe Mitsubishi Pharma, Tsumura, and Yoshitomiyakuhin, and has received research/grant support from Astellas, Daiichi Sankyo, Dainippon Sumitomo Pharma, Eisai, Eli Lilly, Kyowa Hakko Kirin, Mochida Pharmaceutical, MSD, Novartis Pharma, Otsuka Pharmaceutical, Pfizer, Shionogi, and Takeda Pharmaceutical, and is a member of the advisory board of Dainippon Sumitomo Pharma.

${ }^{1}$ Current affiliation: Department of Psychiatry, Hokkaido University Hospital, Sapporo, Japan.

https://doi.org/10.1124/jpet.120.000164.

S This article has supplemental material available at jpet.aspetjournals.org.

\section{Introduction}

Clozapine has superior efficacy against treatment-resistant schizophrenia and causes fewer extrapyramidal symptoms (EPS) compared with other antipsychotic drugs (Leucht et al., 2013). However, clozapine has been reported to cause various adverse events such as agranulocytosis, hypotension, constipation, and sialorrhea (Kishi et al., 2013). Among these, clozapine-induced sialorrhea (CIS) is a frequent adverse reaction that can lead to life-threatening pneumonia due to aspiration (Saenger et al., 2016; Kaplan et al., 2018). Pharmacological approaches that have been recommended for the management of CIS have shown limited efficacy (Bai et al., 2001; Sockalingam et al., 2007; Bird et al., 2011). Hence, it is important to elucidate the expression mechanism of CIS and to develop novel treatment options.

Previous studies on CIS have been conducted at the clinical, in vivo, and in vitro levels. Two clinical studies reported that 
the salivary flow rates of patients on oral clozapine were not different from those of healthy controls (Ben-Aryeh et al., 1996; Rabinowitz et al., 1996). However, another study reported that the salivary flow rate at rest increased 2 to 3 weeks after the start of clozapine treatment (Praharaj et al., 2010). In addition to the discordant results, one common limitation of these clinical studies was that they measured salivary flow rate during daytime, despite the fact that CIS usually occurs during nighttime (Ben-Aryeh et al., 1996; Rabinowitz et al., 1996; Praharaj et al., 2010). Two case reports also suggested the possible role of reduced swallowing function in CIS (McCarthy and Terkelsen, 1994; Pearlman, 1994). However, this hypothesis should be carefully tested in a clinical study since clozapine has the highest risk of sialorrhea and the lowest risk of EPS among antipsychotics (Leucht et al., 2013). Another limitation observed in previous clinical studies is that no studies have focused on the effect of the two major metabolites of clozapine, $N$-desmethylclozapine and clozapine $N$-oxide.

Previous in vivo studies have reported that subcutaneous administration of clozapine has no effect on salivary secretion in both anesthetized and nonanesthetized mice (Ogawa, 1997). On the contrary, intravenous administration of $N$-desmethylclozapine evoked salivary secretion in anesthetized rats (Ekström et al., 2010a; Godoy et al., 2011). As opposed to clozapine, which is a potent antagonist of muscarinic receptor, $N$-desmethylclozapine has agonistic action on muscarinic receptors. The pharmacological profile of $N$-desmethylclozapine is similar to that of clozapine in other respects (Weiner et al., 2004; Rajji et al., 2015). Clozapine $N$-oxide has a less potent action on multiple receptors, including muscarinic receptors (Weiner et al., 2004). This study was noteworthy because the occurrence of sialorrhea was associated with agonistic action on muscarinic receptors, as well as adrenergic receptors (Proctor, 2016; Pedersen et al., 2018). However, there are some limitations in these in vivo experiments from the viewpoint of clinical applicability; most previous studies did not use nonanesthetized animals, and clozapine was not orally administered (Ekström et al., 2010a,b, 2014; Godoy et al., 2011, 2012; Loy et al., 2014). In addition, although it takes at least a few days for the appearance of CIS after clozapine administration in clinical settings, the time lag reported in previous in vivo studies was too short (a minute) (Reinstein et al., 1999; Praharaj et al., 2010). Therefore, a new animal model reflecting the clinical scenario of CIS is warranted.

Previous in vitro studies have shown that clozapine acts on serous and mucous cells of human submandibular salivary gland as a weak muscarinic partial agonist (Riva et al., 2003; Testa Riva et al., 2006). However, another study showed that clozapine acts on rat salivary acinar cells as a muscarinic receptor antagonist (Pochet et al., 2003). In addition to the discordance of the results in clozapine, no previous in vitro studies have yet examined the effects of $N$-desmethylclozapine on the salivary glands.

After reviewing several clinical, in vivo, and in vitro studies on CIS, we hypothesized that $N$-desmethylclozapine is

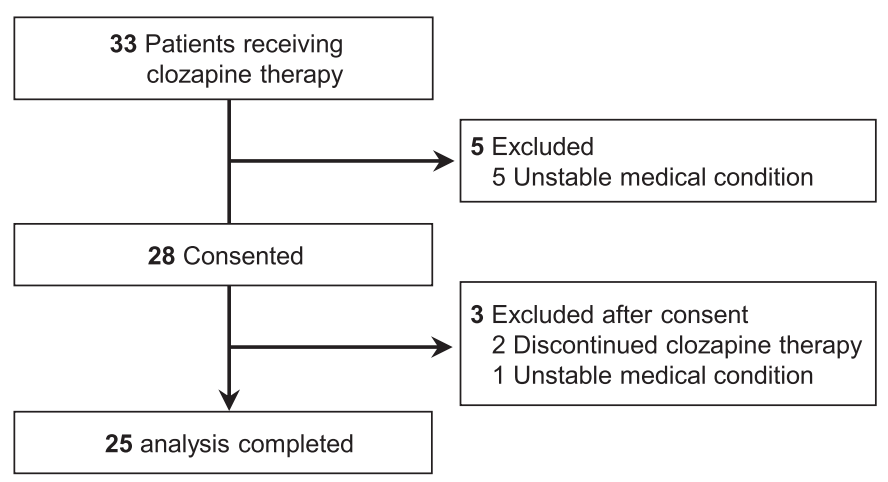

Fig. 1. Flowchart of study participants.

implicated in CIS by increasing salivary secretion via the muscarinic receptor. This is because pharmacological properties of clozapine are not likely to induce sialorrhea nor dysphagia, and several findings in preclinical studies have suggested the involvement of $N$-desmethylclozapine in CIS. To test this hypothesis, we conducted clinical, in vivo, and in vitro studies.

\section{Materials and Methods}

\section{Clinical Study}

Study Design and Participants. A prospective, single-center, observational study was conducted from April 2017 to March 2019. Patients were recruited from Hokkaido University Hospital. A total of 25 patients were enrolled into the study. The inclusion criteria were patients who were 20 years of age or older and diagnosed with schizophrenia by a treating psychiatrists with 5 years or more clinical experience according to the Diagnostic and Statistical Manual of Mental Disorders IV codes diagnosis. The patient must have been receiving stable treatment with clozapine at the time of the study. We excluded patients who were in unstable medical condition or had changed their clozapine dosage within a week. A flowchart of patient recruitment is provided in Figure 1 . The study was approved by the Institutional Review Board of the Hokkaido University Hospital (approval number 016-0141). Written informed consent was obtained from all patients. All studies were conducted in compliance with the Declaration of Helsinki. The study registered with the University Hospital Medical Information Network clinical trial register system (registration number UMIN000026822).

Primary and Secondary Outcomes. The primary outcome was the association between the severity of sialorrhea and serum clozapine or its metabolites concentrations. Secondary outcomes were the association between the severity of sialorrhea and 1) saliva clozapine or its metabolites concentrations, 2) swallowing function, 3) severity of EPS, and 4) patient characteristics.

Outcome Measures. The severity of sialorrhea was assessed using the Drooling Severity and Frequency Scale (DSFS), which is a semiquantitative assessment scale. Two time points were used in assessing the DSFS: daytime (morning, afternoon, and evening) and nighttime (night and asleep at night). To complement the DSFS assessment, a self-administered questionnaire was used that asked about the presence or absence of subjective sialorrhea and, if present, the time in experiencing the most severe sialorrhea. As an objective

ABBREVIATIONS: CIS, clozapine-induced sialorrhea; DIEPSS, Drug-Induced Extrapyramidal Symptom Scale; DSFS, Drooling Severity and Frequency Scale; EPS, extrapyramidal symptoms; HSY cells, human salivary gland cell line; LC-MS/MS, liquid chromatography-tandem mass spectrometry; RSST, Repetitive Saliva Swallowing Test. 
evaluation, unstimulated salivary flow rates were measured by placing a dry cotton in the oral cavity for 2 minutes. The swallowing function was assessed by the Repetitive Saliva Swallowing Test (RSST), and the severity of EPS was assessed through the DrugInduced Extrapyramidal Symptom Scale (DIEPSS).

For the convenience of the patients, all the assessments and data sampling were conducted at the outpatient visit for each patient. The assessment and data sampling of inpatients were conducted on the day of blood collection defined by the clozaril patient monitoring service (Supplemental Methods). Assessment of the RSST and DIEPSS measurements were conducted for all patients. Each patient's data (clinical interviews) were requested within 1 month of giving consent to participate in the study. Unstimulated salivary flow rates and DSFS scores were measured, and self-administered questionnaires were administered every 4 weeks. If clozapine was continued at the same dosage and administration, the interval between assessments and data sampling could be adjusted to a range of 2-12 weeks. The methods used in the study are described in detail in Supplemental Methods. The schedule of the study is shown in Supplemental Table 1.

Sample Collection and Assay. Blood sample was collected after an average of 12 hours (range 3-19 hours) from the last clozapine dose. Saliva sample was collected after an average of 14 hours (range 3-20 hours) from the last clozapine dose. Serum or saliva concentrations of clozapine and its two main metabolites $(N$-desmethylclozapine and clozapine $N$-oxide) were measured by liquid chromatography-tandem mass spectrometry (LC-MS/MS) after liquid-liquid extraction (Wohlfarth et al., 2011). The methods used in the sample collection and assay of clozapine and its metabolites are described in detail in Supplemental Methods.

Sample Size Estimation. A sample size of 25 participants was needed to detect a correlation coefficient of 0.5 with alpha of 0.05 and power of $80 \%$. Since the objective of this clinical study is the identification of strongly positive correlated factors that contribute to CIS, the correlation coefficient was estimated with $r_{\mathrm{s}}=0.5$ (Cohen, 1992). A one-tailed Spearman's correlation was used. We calculated sample size using GPower 3.1.9.2 software (Faul et al., 1992).

Statistical Analyses. A two-tailed Mann-Whitney $U$ test was used to compare the differences in DSFS score of categorical variables (such as sex) between the groups. A one-tailed Spearman's correlation was used to assess the possible correlation between ordered categorical variables (such as DSFS score) and continuous variables (such as serum concentration) or between ordered categorical variables. All data were analyzed by SPSS version 23.0 (IBM Corp., Armonk, NY). All $P$ values for clinical measures were considered significant at $P<0.05$.

\section{Preclinical Study}

Materials. Clozaril (clozapine tablet) for oral administration to the rats were purchased from Novartis Pharma (Tokyo, Japan). Clozapine, $N$-desmethylclozapine, clozapine $N$-oxide, and carbamazepine (internal standard) for LC-MS/MS and cell culture treatment were obtained from Santa Cruz Biotechnology (Santa Cruz, CA). Carbachol and atropine for cell culture treatment were purchased from Sigma-Aldrich (St. Louis, MO).

Animals. Male Wistar rats (6-8 weeks old) weighing $130-200 \mathrm{~g}$ were purchased from Clea Japan Inc. (Tokyo, Japan). All the animals were housed under a 12-hour light/12-hour dark cycle (lights were on from 7:00 to 19:00) and maintained at a temperature of $23 \pm 2^{\circ} \mathrm{C}$ and humidity of $60 \% \pm 10 \%$ in a controlled room. The animals had free access to food and water ad libitum. All animal experiments were conducted according to the guidelines for the Care and Use of Laboratory Animals of Hokkaido University (approval number 17-0005).

Animal Experimental Protocols. One group received a single dose of oral clozapine, whereas the other group received a single daily dose of oral clozapine for 7 days. The dose of clozapine administered to the rats was determined from literature to enable the dose to correspond to a clinically relevant human-equivalent dose (Hou et al.,
2015). Clozapine was prepared by suspending 25,50 , and $100 \mathrm{mg}$ of clozapine tablets in $1 \mathrm{ml}$ water, and a volume of $1 \mathrm{ml} / \mathrm{kg}$ was administered to the rats by oral gavage. The control group received an equivalent volume of water without any clozapine. The body weight of the rats was measured immediately before the administration of clozapine. Salivary secretion was measured four times a day every 6 hours in the single dose treatment group. In contrast, salivary secretion was measured every 24 hours after clozapine administration for 7 days in the repeated dose treatment groups because the salivary volume was maximum at 24 hours after clozapine administration in the single administration trial. Rats were restricted from intake food and water for an hour prior to saliva measurement. The saliva was collected by swiping three preweighed cotton balls in the oral cavity of the rat for 30 seconds. The mass of the saliva was calculated from the change in the mass of the three cotton balls. The method for the saliva collection has been previously described with some modifications (Yamanaka et al., 1985; Nezu et al., 2015).

Assay of Clozapine and Its Metabolites. Blood, submandibular gland, and brain of the rats were collected 24 hours after the single administration of clozapine or 24 hours after the repeated administration of clozapine for 7 days. The extraction procedure and LC-MS/ MS analysis were the same methods as that used in the clinical study (Supplemental Methods).

Cell Culture. Human salivary gland cell line (HSY cells) were kindly provided by Prof. Dr. A. Tanimura (Health Sciences University of Hokkaido, Hokkaido, Japan). The HSY cells were cultured at $37^{\circ} \mathrm{C}$, $5 \% \mathrm{CO}_{2}$ in Dulbecco's modified Eagle's medium supplemented with $10 \%$ fetal bovine serum, $100 \mathrm{U} / \mathrm{ml}$ penicillin, and $100 \mu \mathrm{g} / \mathrm{ml}$ streptomycin as previously described (Yamada et al., 2010).

Calcium Assays in Human Salivary Gland Cell Line. Intracellular $\mathrm{Ca}^{2+}$ mobilization was measured in triplicate in HSY cells using fura-2 AM (Dojindo Laboratory, Kumamoto, Japan), and fura-2 fluorescence was measured by an Infinite M200 microplate reader (TECAN, Kanagawa, Japan) as previously described (Nezu et al., 2015) with several modifications (Supplemental Methods).

\section{Statistical Analyses}

We used two-tailed $t$ tests or one-way ANOVA to compare the differences of continuous variables. ANOVA followed by Tukey's post hoc test was performed. A $P$ value $<0.05$ was considered as statistically significant. All analyses were performed with SPSS version 23.0 (IBM Corp). The data were presented as means \pm S.D.

\section{Results}

\section{Clinical Study Results}

Demographic and Clinical Characteristics. A total of 25 patients with schizophrenia participated in this study. Demographic and clinical characteristics of patients are listed in Table 1. No significant association were observed between clinical characteristics (age, sex, body weight, daily clozapine dose, duration of clozapine treatment, smoking and drinking caffeine habits, comedication with other antipsychotics, benzodiazepines, and anticholinergic agents) and diurnal or nocturnal DSFS score (Supplemental Tables 2 and 3).

Association Between Severity of Sialorrhea and Serum or Saliva Clozapine and Its Metabolites Concentrations. Serum $\left(r_{\mathrm{s}}=0.453, P=0.012\right)$ and saliva $\left(r_{\mathrm{s}}=\right.$ $0.340, P=0.048$ ) concentrations of $N$-desmethylclozapine were significantly correlated with "nocturnal" DSFS score but not "diurnal" DSFS score (Fig. 2, A and D; Supplemental Fig. 1, A and D). On the contrary, neither serum nor saliva concentrations of clozapine and clozapine $N$-oxide were correlated with nocturnal or diurnal DSFS scores (Fig. 2, B, C, E, and F; 
TABLE 1

Clinical and demographic characteristics

\begin{tabular}{lc}
\hline Clinical characteristics $(n=25)$ & \\
\hline Age, yr, mean (S.D.) [range] & $36.6(10.5)[24-70]$ \\
Male sex, $n$ (\%) & $9(36)$ \\
Weight, kg, mean (S.D.) [range] & $63.3(13.1)[45-88]$ \\
Daily clozapine dose, mg, mean & $381.0(139.3)[125-600]$ \\
$\quad$ (S.D.) [range] & \\
Duration of clozapine treatment, & $1285.9(922.2)[246-2960]$ \\
$\quad$ day, mean (S.D.) [range] & $4(16)$ \\
Smoker, $n$ (\%) & $10(40)$ \\
Caffeine drinker, $n(\%)$ & \\
Co-medication, $n(\%)$ & $3(12)$ \\
Antipsychotics & $16(64)$ \\
Benzodiazepines & $6(24)$ \\
Anticholinergic agents &
\end{tabular}

Supplemental Fig. 1, B, C, E, and F). Nocturnal DSFS score was significantly higher than diurnal DSFS score (Fig. 3A). Diurnal DSFS score was significantly correlated with diurnal salivary flow rates (Supplemental Fig. 2).

Fluctuation of Expression and Severity of Sialorrhea. From the results of the self-administered questionnaire, $92 \%$ of the patients reported that they experienced sialorrhea. Most patients experienced sialorrhea while asleep at night regardless of the clozapine dosage and dosing frequency (Fig. 3B; Supplemental Figs. 3 and 4).

Association Between Severity of Sialorrhea and Swallowing Function or Severity of Extrapyramidal Symptom. There were no significant correlations between the RSST score and diurnal DSFS score $\left(r_{\mathrm{s}}=0.021, P=0.461\right)$, nocturnal DSFS score $\left(r_{\mathrm{s}}=-0.168, P=0.211\right)$, or diurnal salivary flow rates $\left(r_{\mathrm{s}}=0.282, P=0.086\right.$ ) (Supplemental Fig. 5). In the RSST score, none of our patients showed a swallowing ability of less than three times, which would have been an indication of dysphagia (Supplemental Fig. 5). Similarly, DIEPSS score was not correlated with diurnal DSFS score $\left(r_{\mathrm{s}}=0.173, P=0.203\right)$, nocturnal DSFS score $\left(r_{\mathrm{s}}=0.160, P=0.223\right)$, or diurnal salivary flow rates $\left(r_{\mathrm{s}}=\right.$ $0.212, P=0.155$ ) (Supplemental Fig. 6). The RSST and DIEPSS scores were not related to clozapine dose or to clozapine and its metabolites' concentrations in the serum and saliva (Supplemental Figs. 7 and 8).

\section{Preclinical Results (In Vivo Research)}

Effects of Oral Administration of Clozapine on Salivary Secretion. A single dose administration of clozapine did not significantly increase salivary secretion compared with that of the control group regardless of the clozapine dose (Supplemental Fig. 9; Supplemental Results). In the repeated administration trials, a dose of $25 \mathrm{mg} / \mathrm{kg}$ clozapine did not significantly increase salivation as compared with controls in the total time course (Fig. 4). A dose of $50 \mathrm{mg} / \mathrm{kg}$ clozapine significantly induced more salivary secretions compared with that in the control on the fourth, sixth, and seventh day of administration, and a dose of $100 \mathrm{mg} / \mathrm{kg}$ significantly induced more salivary secretions from days 3 to 7 (Fig. 4). Administration of $100 \mathrm{mg} / \mathrm{kg}$ clozapine significantly increased salivation on the seventh day after administration compared with
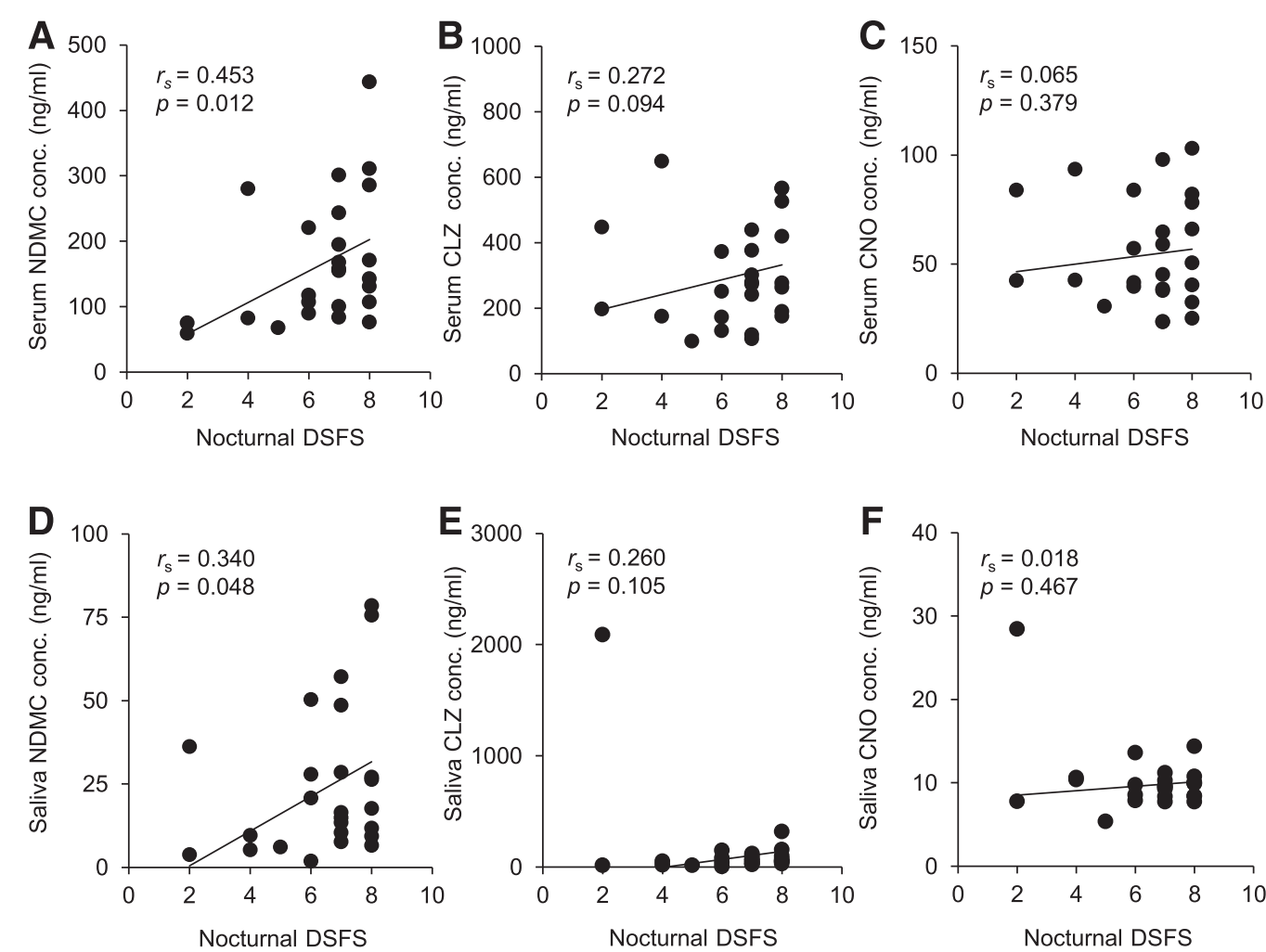

Fig. 2. Serum and saliva concentrations of clozapine and its metabolites were correlated with nocturnal DSFS scores. The Spearman rank correlation test was applied to find the association between $N$-desmethylclozapine (NDMC) (A), clozapine (CLZ) (B), and clozapine $N$-oxide (CNO) (C) concentrations in serum and nocturnal DSFS score. The Spearman rank correlation test was applied to find the association between NDMC (D), CLZ (E), and CNO (F) concentrations in saliva and nocturnal DSFS scores. One circle indicates the result per patient. The solid line indicates correlation coefficient, $r_{\mathrm{s}}$. Significance was set at $* P<0.05$. 

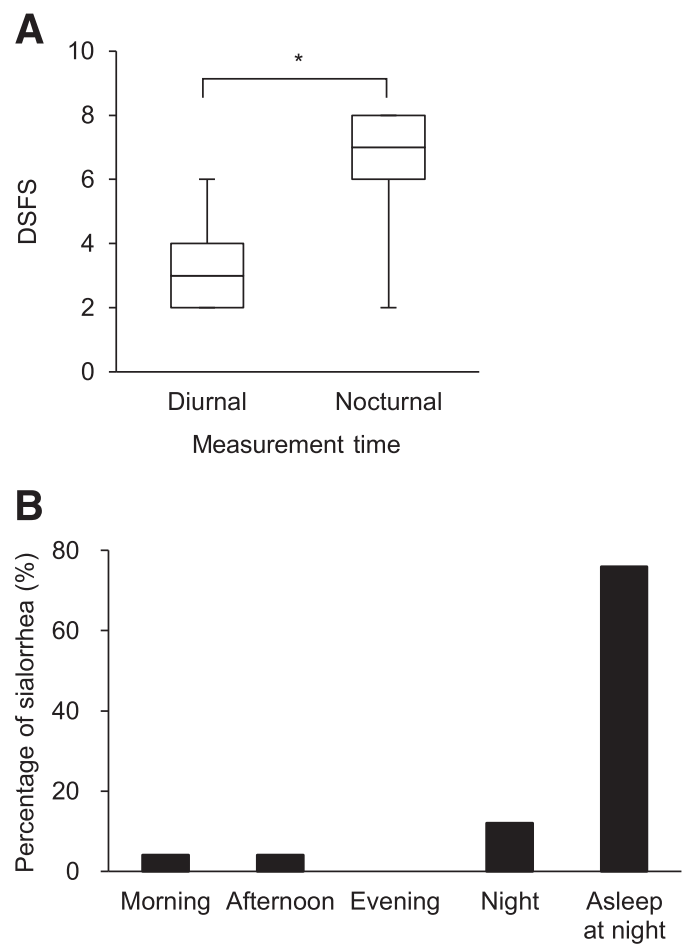

Fig. 3. Intraday fluctuations of expression and severity of sialorrhea. (A) Comparison of diurnal and nocturnal DSFS scores. (B) Comparison of incidence rates of CIS at different time points.

the administration of other doses (Fig. 4G). The body weights were not statistically different among the groups throughout the treatment period (Supplemental Fig. 10).
Serum and Tissues Concentrations of Clozapine and Its Metabolites in the Animal Model. We measured concentrations of clozapine and its metabolites in the serum, submandibular gland, and brain 24 hours after the single and repeated oral administrations of clozapine $(100 \mathrm{mg} / \mathrm{kg})$. The mean serum concentration of clozapine in the repeated administration group (clozapine $100 \mathrm{mg} / \mathrm{kg}$ for 7 days) was $27.8 \pm 10.8 \mathrm{ng} / \mathrm{ml}$, which was in line with the clinically estimated serum concentration of clozapine in humans (Fig. 5A). The concentration of $N$-desmethylclozapine in the repeated administration group was significantly higher than those in the single administration group, regardless of the assay matrix (Fig. 5). In the repeated administration group, the concentrations of $N$-desmethylclozapine were higher than those of clozapine and clozapine $\mathrm{N}$-oxide in the serum and submandibular gland (Fig. 5, A and B); however, there were no significant differences between the concentration of $N$-desmethylclozapine and that of clozapine in the brain (Fig. 5C).

\section{Preclinical Results (In Vitro Research)}

Effects of Clozapine and Its Metabolites on $\mathrm{Ca}^{2+}$ Responses in Human Salivary Gland Cell Line. We examined the effect of clozapine and its metabolites on intracellular $\mathrm{Ca}^{2+}$ concentration in fura-2-loaded HSY cells. In this experiment, treatment with $100 \mu \mathrm{M} \mathrm{N}$-desmethylclozapine induced a significant increase in intracellular $\mathrm{Ca}^{2+}$ concentration (Fig. 6A); however, such an increase in concentration was not observed in treatments with $100 \mu \mathrm{M}$ clozapine or $100 \mu \mathrm{M}$ clozapine $N$-oxide (Fig. 6, B and C). Also, $N$-desmethylclozapine induced increase in intracellular
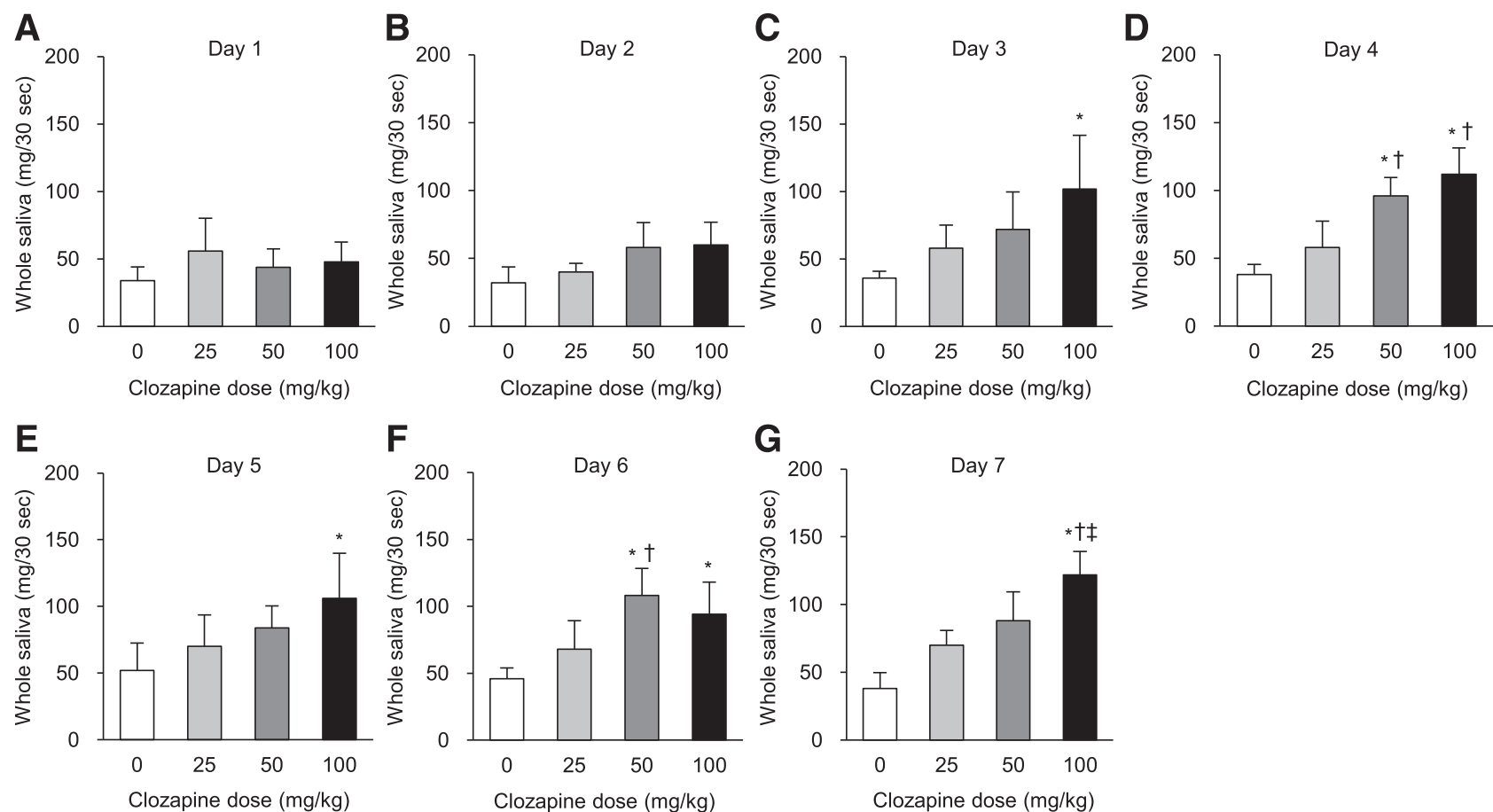

Fig. 4. Effects of repeated oral administration of clozapine on salivary secretion in rats. Vehicle and clozapine $(25,50,100 \mathrm{mg} / \mathrm{kg}) \mathrm{were}$ administered daily to rats for 7 days. Whole saliva was measured every 24 hours after oral administration of vehicle or several clozapine doses 1 day (A), 2 days (B), 3 days $(\mathrm{C}), 4$ days $(\mathrm{D}), 5$ days $(\mathrm{E}), 6$ days $(\mathrm{F})$, and 7 days $(\mathrm{G})$ after administration. Each column represents the mean with S.D. of five determinations. ${ }^{*} P<0.05$ vs. the control; ${ }^{\dagger} P<0.05$ vs. clozapine at $25 \mathrm{mg} / \mathrm{kg} ;{ }^{\ddagger} P<0.05$ vs. clozapine at $50 \mathrm{mg} / \mathrm{kg}$. 
A

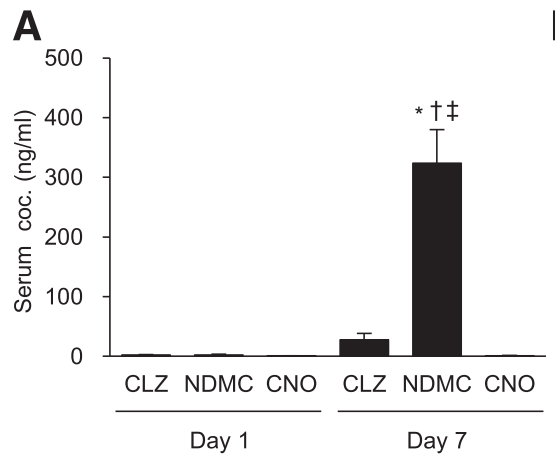

B

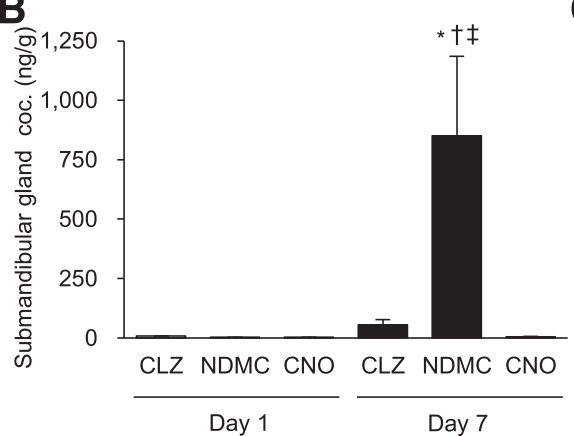

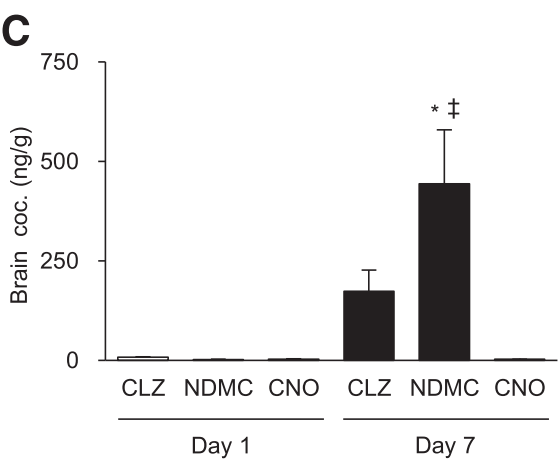

Fig. 5. Serum, submandibular gland, and brain concentrations of clozapine and its metabolites after a single or repeated 7 days oral administration of clozapine. (A) Serum concentrations of clozapine (CLZ), $N$-desmethylclozapine (NDMC), and clozapine $N$-oxide (CNO) were measured 24 hours after a single or seventh oral administration of clozapine $100 \mathrm{mg} / \mathrm{kg}$. (B) Submandibular gland concentrations of CLZ, NDMC, and CNO were measured 24 hours after a single or seventh oral administration of clozapine $100 \mathrm{mg} / \mathrm{kg}$. (C) Brain concentrations of CLZ, NDMC, and CNO were measured 24 hours after a single or seventh oral administration of clozapine $100 \mathrm{mg} / \mathrm{kg}$. Each column represents the mean with S.D. of three determinations. ${ }^{*} P<0.05$; significantly different from a single oral administration group. ${ }^{\dagger} P<0.05$; significantly different from CLZ concentration in repeated oral administration group. ${ }^{\ddagger} P<0.05$; significantly different from CNO concentration in repeated oral administration group.

$\mathrm{Ca}^{2+}$ concentration was inhibited by pretreating the cells with $1 \mu \mathrm{M}$ atropine (Fig. 6D).

\section{Discussion}

In our clinical study, we found that high concentrations of $N$-desmethylclozapine, but not those of clozapine and clozapine $N$-oxide, were associated with CIS. This result can be explained by the differences in the pharmacological properties between clozapine and its metabolites. The salivary secretion is induced mainly by cholinergic stimulation (Bymaster et al., 2003; Nakamura et al., 2004). Clozapine or clozapine $N$-oxide is less potent in agonist acting on muscarinic receptors (Weiner et al., 2004). In contrast, $N$-desmethylclozapine has a partial agonistic activity at muscarinic receptors (Weiner et al., 2004). Thus, the pharmacological properties of $N$-desmethylclozapine is more advantageous in inducing salivary secretions than clozapine or clozapine $N$-oxide. Previous in vivo study has reported that the administration of $N$-desmethylclozapine, alone or in combination with clozapine, increased the flow of saliva compared with the administration of clozapine alone (Godoy et al., 2011).
The same study also reported that $N$-desmethylclozapine affects the salivary flow rates in a concentration-dependent manner (Godoy et al., 2011). All these studies support the hypothesis that $N$-desmethylclozapine is more important as a causative agent of CIS than clozapine.

Our clinical study showed that nocturnal DSFS score was significantly higher than diurnal DSFS score and that serum and saliva $N$-desmethylclozapine concentrations were significantly correlated with nocturnal but not diurnal DSFS score. Secreted saliva is swallowed periodically and unconsciously during waking hours (Lear et al., 1965; Dent et al., 1980). However, the frequency of salivary swallowing is markedly reduced during sleep (Lear et al., 1965; Lichter and Muir, 1975; Dent et al., 1980). Similarly, the salivary secretion by the salivary glands is reduced during sleep (Thie et al., 2002; Proctor, 2016). Therefore, saliva rarely spills from the oral cavity during sleep. We assume that the cholinergic activity of $N$-desmethylclozapine is not sufficient to increase saliva production during the day. However, during sleep at night, endogenous saliva production and frequency of saliva swallowing is markedly reduced, thereby revealing the cholinergic activity of $N$-desmethylclozapine.
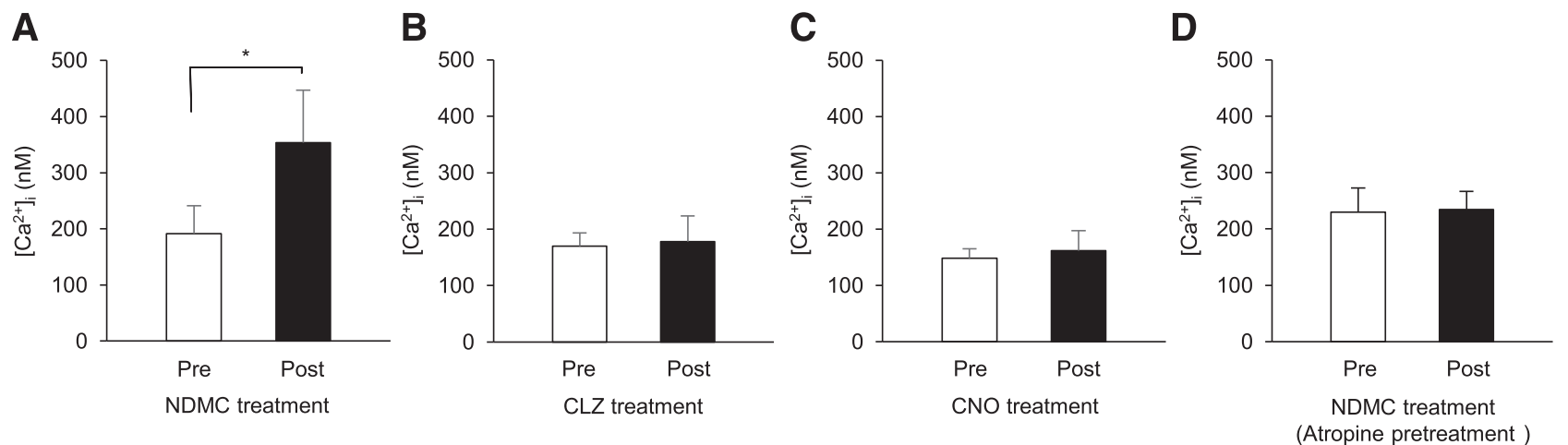

Fig. 6. Effects of clozapine and its metabolites on $\left[\mathrm{Ca}^{2+}\right]_{i}$ in human salivary gland cell line $100 \mu \mathrm{M} N$-desmethylclozapine (NDMC). (A), $100 \mu \mathrm{M}$ clozapine (CLZ) (B), and $100 \mu \mathrm{M}$ clozapine $N$-oxide (CNO) (C) induced increase in intracellular $\mathrm{Ca}^{2+}$ concentration ([Ca $\left.{ }^{2+}\right]_{\mathrm{i}}$ ) in $\mathrm{HSY}$ cells. (D) Inhibitory effect of pretreatment with $1 \mu \mathrm{M}$ atropine on the NDMC induced increase in $\left[\mathrm{Ca}^{2+}\right]_{i}$. Each column represents means with S.D. of three to five determinations. Significance was set at $* P<0.05$. 
In our in vivo study, we succeeded in developing a new animal model that reflects CIS in clinical conditions. Our CIS animal model differs from previous ones in two ways: 1) in our animal model, there was oral and repeated administration of clozapine; however, previous animal models administered clozapine or $N$-desmethylclozapine parenterally in a single dose; and 2) in our animal model, we measured the amount of saliva in a conscious state because we developed a new methodology for collecting saliva from rats in unanesthetized state. Our animal model began showing increased salivation more than 3 days after the repeated oral administration of clozapine, whereas a previous animal model did not show increased salivation by a single subcutaneous administration of clozapine (Ogawa, 1997). The onset of CIS in our new model was consistent with CIS in clinical settings, which occurs at least a few days after the start of clozapine administration (Reinstein et al., 1999; Praharaj et al., 2010).

The therapeutic reference range of clozapine is $350-600 \mathrm{ng} / \mathrm{ml}$ (Urban and Cubała, 2017; Hiemke et al., 2018). This range is based on the assumption that clozapine is administered twice a day. In a clinical setting, clozapine serum trough level, which was measured 24 hours after once daily clozapine doses in the same manner as in our in vivo study, was approximately $70 \mathrm{ng} / \mathrm{ml}$ (Menkes et al., 2018). In our in vivo study, serum trough level of our animal model, which was measured 24 hours after repeated administration of clozapine for 7 days, was approximately $30 \mathrm{ng} / \mathrm{ml}$; these trough levels were roughly in agreement.

Furthermore, in our animal model, we found that the serum and submandibular gland concentrations of $N$-desmethylclozapine were higher than those of clozapine on day 7 . This result suggests that high concentrations of $\mathrm{N}$-desmethylclozapine in the serum and submandibular gland of conscious rats due to repeated oral administration of clozapine can cause salivation. Thus, the observed contribution of $N$-desmethylclozapine to salivary secretion in our in vivo study is consistent with the results of our clinical and previous in vivo studies (Ekström et al., 2010a; Godoy et al., 2011).

Our in vitro study demonstrated that $N$-desmethylclozapine but not clozapine or clozapine $N$-oxide induced $\mathrm{Ca}^{2+}$ responses in HSY cells and that this effect was inhibited by atropine. To the best of our knowledge, this is the first study that showed the effect of $N$-desmethylclozapine on $\mathrm{Ca}^{2+}$ responses in the human salivary gland cell line. Strong correlations of $N$-desmethylclozapine with "nocturnal" DSFS score and salivary secretions were also showed in the clinical study and in animal model, respectively. Together with these results, the present study suggests that salivations in clozapine-treated patients and rats were induced by the effects of $N$-desmethylclozapine on the muscarinic receptors. However, we observed no significant difference in DSFS scores between anticholinergic users and nonusers in our clinical study. Anticholinergic drug users were taking biperiden or scopolamine butylbromide, which are reportedly effective against CIS (Liang et al., 2010; Takeuchi et al., 2015). This result may be attributed to the fact that most anticholinergic drug users took the drug during the day, which might have been less effective against nocturnal sialorrhea.

It has been known that salivary gland cells express two subtypes of muscarinic receptors, $\mathrm{M}_{1}$ and $\mathrm{M}_{3}$, and the salivary secretions are mainly regulated by $\mathrm{M}_{3}$ receptor (Nakamura et al., 2004). The HSY cells used in our study are also express muscarinic receptor subtypes $\mathrm{M}_{1}$ and $\mathrm{M}_{3}$ (Lin et al., 2008). Clozapine, which possesses a weak agonistic action at the $\mathrm{M}_{1}$ receptor, failed to evoke the $\mathrm{Ca}^{2+}$ response in HSY cells. This in vitro study suggests that the partial agonistic action of $N$-desmethylclozapine at the $\mathrm{M}_{3}$ receptor could be sufficient to induced $\mathrm{Ca}^{2+}$ response and provides a possibility for inducing salivation through the $\mathrm{M}_{3}$ receptor mediated $\mathrm{Ca}^{2+}$ responses in salivary cells in patients and rats.

There are several limitations in our clinical study. First, due to the small sample size, we could not adjust for confounding factors relevant to CIS, and we only examined simple correlations. Possible confounding factors that might be associated with serum clozapine and its metabolites concentrations include the following: age, sex, body weight, daily clozapine dose, duration of clozapine treatment, and smoking and caffeine drinking habits (Haring et al., 1989; Perry et al., 1998). Moreover, comedication with other antipsychotics, benzodiazepines, and anticholinergic agents are also confounding factors related to the severity of sialorrhea (Freudenreich, 2005; Miranda-Rius et al., 2009). However, these possible confounding factors may have had only a limited effect on this study, if any, because they were not correlated and differed with DSFS scores (Supplemental Tables 2 and 3). Second, we did not have a scheduled time for drug administration and blood or saliva sampling because most of our subjects were outpatients. This might affect the association between clozapine dose and its serum and saliva concentrations, although the correlation coefficient between them in our study (Supplemental Fig. 11) was similar to that reported in previous studies (Haring et al., 1989; Palego et al., 2002; Wohkittel et al., 2016). In addition, we could not assess swallowing function and salivary flow rate while the patients were asleep at night, the time when CIS occurred. The lack of these data to determine the association between $N$-desmethylclozapine concentration and CIS is a major limitation of our in vivo and in vitro study.

We propose that our new CIS animal model will serve as a bridge between basic and clinical research. For example, it could be helpful to examine the activations or the alterations in gene and protein expression of several receptors, transporters, and channels, such as $\mathrm{M}_{3}$ receptor, $\mathrm{Na}^{+}-\mathrm{K}^{+}-2 \mathrm{Cl}^{-}$ cotransporter, and aquaporin-5 on the salivary gland to elucidate the mechanisms of delayed onset of CIS. A previous study showed that gene and protein expression of the $\mathrm{M}_{3}$ receptor increased in the salivary glands after a continuous administration of muscarinic agonist compared with a single administration (Minagi et al., 2018). Furthermore, there is a possibility that clozapine may cause sialorrhea by direct action on the central nervous system to regulate salivary secretions, since it shows a high intracerebral permeability after oral administration (Borella et al., 2008; Proctor, 2016). For this reason, it is necessary to examine these hypotheses by an autonomic ganglionic blocker such as a hexamethoniumtreated CIS animal model in further studies.

In conclusion, we found an association between nocturnal sialorrhea and serum and saliva concentrations of $N$-desmethylclozapine in our clinical study. In our in vivo study, delayed-onset salivation increments and high serum and submandibular gland concentrations of $\mathrm{N}$-desmethylclozapine were observed after repeated oral administration of clozapine in our new CIS animal model. In our in vitro study, only $N$-desmethylclozapine induced $\mathrm{Ca}^{2+}$ responses via the muscarinic receptor in human 
salivary gland. These results suggest that $N$-desmethylclozapine is implicated in CIS by increasing nocturnal salivation via the muscarinic receptor. Our findings provide important knowledge that can lead to the development of an effective novel treatment option for CIS, such as the identification of a new candidate drug and the determination of its optimum administration time. Our studies have laid the first step toward improving the CIS outcome.

\section{Acknowledgments}

We would like to thank Editage (http://www.editage.jp) for the English language editing.

\section{Authorship Contributions}

Participated in research design: Ishikawa, Kobayashi, Hashimoto,

Tanimura, Kusumi, Iseki.

Conducted experiments: Ishikawa, Mikami.

Performed data analysis: Ishikawa.

Wrote or contributed to the writing of the manuscript: Ishikawa, Kobayashi, Hashimoto, Tanimura, Narumi, Furugen, Kusumi, Iseki.

\section{References}

Bai YM, Lin CC, Chen JY, and Liu WC (2001) Therapeutic effect of pirenzepine for clozapine-induced hypersalivation: a randomized, double-blind, placebo-controlled, cross-over study. J Clin Psychopharmacol 21:608-611.

Ben-Aryeh H, Jungerman T, Szargel R, Klein E, and Laufer D (1996) Salivary flowrate and composition in schizophrenic patients on clozapine: subjective reports and laboratory data. Biol Psychiatry 39:946-949.

Bird AM, Smith TL, and Walton AE (2011) Current treatment strategies for clozapine-induced sialorrhea. Ann Pharmacother 45:667-675.

Borella TL, De Luca LA Jr., Colombari DS, and Menani JV (2008) Central muscarinic receptor subtypes involved in pilocarpine-induced salivation, hypertension and water intake. Br J Pharmacol 155:1256-1263.

Bymaster FP, Carter PA, Yamada M, Gomeza J, Wess J, Hamilton SE, Nathanson NM, McKinzie DL, and Felder CC (2003) Role of specific muscarinic receptor subtypes in cholinergic parasympathomimetic responses, in vivo phosphoinositide hydrolysis, and pilocarpine-induced seizure activity. Eur J Neurosci 17: 1403-1410.

Cohen J (1992) A power primer. Psychol Bull 112:155-159.

Dent J, Dodds WJ, Friedman RH, Sekiguchi T, Hogan WJ, Arndorfer RC, and Petrie DJ (1980) Mechanism of gastroesophageal reflux in recumbent asymptomatic human subjects. $J$ Clin Invest 65:256-267.

Ekström J, Godoy T, Loy F, and Riva A (2014) Parasympathetic vasoactive intestinal peptide (VIP): a likely contributor to clozapine-induced sialorrhoea. Oral Dis 20: e90-e96.

Ekström J, Godoy T, and Riva A (2010a) $N$-Desmethylclozapine exerts dual and opposite effects on salivary secretion in the rat. Eur J Oral Sci 118:1-8.

Ekström J, Godoy T, and Riva A (2010b) Clozapine: agonistic and antagonistic salivary secretory actions. $J$ Dent Res 89:276-280.

Faul F, Erdfelder E, Lang AG, and Buchner A (2007) G*Power 3: a flexible statistical power analysis program for the social, behavioral, and biomedical sciences. Behav Res Methods 39:175-191.

Freudenreich O (2005) Drug-induced sialorrhea. Drugs Today (Barc) 41:411-418.

Godoy T, Riva A, and Ekström J (2011) Clozapine-induced salivation: interaction with $N$-desmethylclozapine and amisulpride in an experimental rat model. Eur J Oral Sci 119:275-281.

Godoy T, Riva A, and Ekström J (2012) Atypical antipsychotics--effects of amisulpride on salivary secretion and on clozapine-induced sialorrhea. Oral Dis 18 $680-691$.

Haring C, Meise U, Humpel C, Saria A, Fleischhacker WW, and Hinterhuber H (1989) Dose-related plasma levels of clozapine: influence of smoking behaviour, sex and age. Psychopharmacology (Berl) 99 (Suppl):S38-S40.

Hiemke C, Bergemann N, Clement HW, Conca A, Deckert J, Domschke K, Eckermann G, Egberts K, Gerlach M, Greiner C, et al. (2018) Consensus guidelines for therapeutic drug monitoring in neuropsychopharmacology: Update 2017. Pharmacopsychiatry 51:9-62.

Hou ML, Lin CH, Lin LC, and Tsai TH (2015) The drug-drug effects of rhein on the pharmacokinetics and pharmacodynamics of clozapine in rat brain extracellular fluid by in vivo microdialysis. J Pharmacol Exp Ther 355:125-134.

Kaplan J, Schwartz AC, and Ward MC (2018) Clozapine-associated aspiration pneumonia: case series and review of the literature Psychosomatics 59:199-203.

Kishi T, Fujita K, Furukawa O, Suzuki T, Moriwaki M, Nitta M, Hattori M, Tsunoka T, Chekuri R, Kane JM, et al. (2013) Efficacy and tolerability of clozapine in Japanese patients with treatment-resistant schizophrenia: results from a 12 -week, flexible dose study using raters masked to antipsychotic choice. Asian J Psychiatr 6:200-207.

Lear CSC, Flanagan JB Jr., and Moorrees CFA (1965) The frequency of deglutition in man. Arch Oral Biol 10:83-100.

Leucht S, Cipriani A, Spineli L, Mavridis D, Orey D, Richter F, Samara M, Barbui C, Engel RR, Geddes JR, et al. (2013) Comparative efficacy and tolerability of 15 antipsychotic drugs in schizophrenia: a multiple-treatments meta-analysis. Lancet 382:951-962
Liang CS, Ho PS, Shen LJ, Lee WK, Yang FW, and Chiang KT (2010) Comparison of the efficacy and impact on cognition of glycopyrrolate and biperiden for clozapineinduced sialorrhea in schizophrenic patients: a randomized, double-blind, crossover study. Schizophr Res 119:138-144.

Lichter I and Muir RC (1975) The pattern of swallowing during sleep. Electroencephalogr Clin Neurophysiol 38:427-432.

Lin AL, Zhu B, Zhang W, Dang H, Zhang BX, Katz MS, and Yeh CK (2008) Distinct pathways of ERK activation by the muscarinic agonists pilocarpine and carbachol in a human salivary cell line. Am J Physiol Cell Physiol 294: C1454-C1464.

Loy F, Isola M, Isola R, Lilliu MA, Solinas P, Conti G, Godoy T, Riva A, and Ekström $J$ (2014) The antipsychotic amisulpride: ultrastructural evidence of its secretory activity in salivary glands. Oral Dis 20:796-802.

McCarthy RH and Terkelsen KG (1994) Esophageal dysfunction in two patients after clozapine treatment. J Clin Psychopharmacol 14:281-283.

Menkes DB, Glue P, Gale C, Lam F, Hung CT, and Hung N (2018) Steady-state clozapine and norclozapine pharmacokinetics in Maori and European patients. EBioMedicine 27:134-137.

Minagi HO, Ikai K, Araie T, Sakai M, and Sakai T (2018) Benefits of long-term pilocarpine due to increased muscarinic acetylcholine receptor 3 in salivary glands. Biochem Biophys Res Commun 503:1098-1102.

Miranda-Rius J, Brunet-Llobet L, Lahor-Soler E, and Farré M (2009) Drug-induced sialorrhoea and excessive saliva accumulation. Prescrire Int 18:119-121.

Nakamura T, Matsui M, Uchida K, Futatsugi A, Kusakawa S, Matsumoto N, Nakamura K, Manabe T, Taketo MM, and Mikoshiba K (2004) $\mathrm{M}_{3}$ muscarinic acetylcholine receptor plays a critical role in parasympathetic control of salivation in mice. $J$ Physiol 558:561-575.

Nezu A, Morita T, Tojyo Y, Nagai T, and Tanimura A (2015) Partial agonistic effects of pilocarpine on $\mathrm{Ca}^{2+}$ responses and salivary secretion in the submandibular glands of live animals. Exp Physiol 100:640-651.

Ogawa T (1997) The paradoxical effects of the dibenzodiazepine derivative, clozapine, on autonomic stimulating drug-induced salivary responses in mice. Jpn J Oral Biol 39.284-296.

Palego L, Biondi L, Giannaccini G, Sarno N, Elmi S, Ciapparelli A, Cassano GB, Lucacchini A, Martini C, and Dell'Osso L (2002) Clozapine, norclozapine plasma levels, their sum and ratio in 50 psychotic patients: influence of patient-related variables. Prog Neuropsychopharmacol Biol Psychiatry 26 $473-480$

Pearlman C (1994) Clozapine, nocturnal sialorrhea, and choking. J Clin Psychopharmacol 14:283.

Pedersen AML, Sørensen CE, Proctor GB, Carpenter GH, and Ekström J (2018) Salivary secretion in health and disease. J Oral Rehabil 45:730-746.

Perry PJ, Bever KA, Arndt S, and Combs MD (1998) Relationship between patient variables and plasma clozapine concentrations: a dosing nomogram. Biol Psychiatry 44:733-738.

Pochet S, García-Marcos M, Fernandez M, Marino A, and Dehaye JP (2003) Regulation by clozapine of calcium handling by rat submandibular acinar cells. Cell Calcium 34:465-475.

Praharaj SK, Jana AK, Goswami K, Das PR, Goyal N, and Sinha VK (2010) Salivary flow rate in patients with schizophrenia on clozapine. Clin Neuropharmacol 33: 176-178.

Proctor GB (2016) The physiology of salivary secretion. Periodontol $2000 \mathbf{7 0}$ : 11-25.

Rabinowitz T, Frankenburg FR, Centorrino F, and Kando J (1996) The effect of clozapine on saliva flow rate: a pilot study. Biol Psychiatry 40:1132-1134.

Rajji TK, Mulsant BH, Davies S, Kalache SM, Tsoutsoulas C, Pollock BG, and Remington G (2015) Prediction of working memory performance in schizophrenia by plasma ratio of clozapine to $N$-desmethylclozapine. Am J Psychiatry 172:579-585.

Reinstein MJ, Sirotovskaya LA, Chasanov MA, Jones LE, and Mohan S (1999) Comparative efficacy and tolerability of benzatropine and terazosin in the treatment of hypersalivation secondary to clozapine. Clin Drug Investig 17 97-102.

Riva A, Puxeddu R, Loy F, Isola M, Cabras T, and Testa Riva F (2003) Serous and mucous cells of human submandibular salivary gland stimulated in vitro by isoproterenol, carbachol and clozapine: an LM, TEM, and HRSEM Study. Eur J Morphol 41:83-87.

Saenger RC, Finch TH, and Francois D (2016) Aspiration pneumonia due to clozapine-induced sialorrhea. Clin Schizophr Relat Psychoses 9:170-172.

Sockalingam S, Shammi C, and Remington G (2007) Clozapine-induced hypersalivation: a review of treatment strategies. Can J Psychiatry 52: 377-384.

Takeuchi I, Suzuki T, Kishi T, Kanamori D, Hanya M, Uno J, Fujita K, and Kamei $\mathrm{H}$ (2015) Effect of scopolamine butylbromide on clozapine-induced hypersalivation in schizophrenic patients: a case series. Clin Psychopharmacol Neurosci 13: $109-112$

Testa Riva F, Puxeddu R, Loy F, Conti G, and Riva A (2006) Cytomorphological study on human submandibular gland following treatment with secretagogue drugs. Cell Tissue Res 324:347-352.

Thie NM, Kato T, Bader G, Montplaisir JY, and Lavigne GJ (2002) The significance of saliva during sleep and the relevance of oromotor movements. Sleep Med Rev 6 : $213-227$.

Urban AE and Cubała WJ (2017) Therapeutic drug monitoring of atypical antipsychotics. Psychiatr Pol 51:1059-1077.

Weiner DM, Meltzer HY, Veinbergs I, Donohue EM, Spalding TA, Smith TT, Mohell N, Harvey SC, Lameh J, Nash N, et al. (2004) The role of $\mathrm{M}_{1}$ muscarinic receptor agonism of $N$-desmethylclozapine in the unique clinical effects of clozapine. Psychopharmacology (Berl) 177:207-216.

Wohkittel C, Gerlach M, Taurines R, Wewetzer C, Unterecker S, Burger R, Schreck D, Mehler-Wex C, Romanos M, and Egberts K (2016) Relationship between 
clozapine dose, serum concentration, and clinical outcome in children and adolescents in clinical practice. J Neural Transm (Vienna) 123:1021-1031.

Wohlfarth A, Toepfner N, Hermanns-Clausen M, and Auwärter V (2011) Sensitive quantification of clozapine and its main metabolites norclozapine and clozapine- $N$ oxide in serum and urine using LC-MS/MS after simple liquid-liquid extraction work-up. Anal Bioanal Chem 400:737-746.

Yamada T, Ryo K, Tai Y, Tamaki Y, Inoue H, Mishima K, Tsubota K, and Saito I (2010) Evaluation of therapeutic effects of astaxanthin on impairments in salivary secretion. J Clin Biochem Nutr 47:130-137.
Yamanaka S, Suyama Y, and Nishimura M (1985) Study on salivary secretion in organophosphorus poisoning: relation to cholinesterase inhibition in blood and organs of rats. $J$ Dent Health 35:203-212.

Address correspondence to: Masaki Kobayashi, Laboratory of Clinical Pharmaceutics and Therapeutics, Division of Pharmasciences, Faculty of Pharmaceutical Sciences, Hokkaido University, Kita-12-jo, Nishi-6-chome, Kita-ku, Sapporo 060-0812, Japan. E-mail: masaki@pharm.hokudai.ac.jp 